\title{
PARASITOIDES ASOCIADOS A LOS ENROLLADORES DE HOJAS DE ZARZAMORA ARGYROTAENIA MONTEZUMAE WALSINGHAM Y AMORBIA SP. (LEPIDOPTERA: TORTRICIDAE), EN MICHOACÁN, MÉXICO
}

\author{
Ana Mabel MARTÍNEZ, ${ }^{1}$ ORNella BARRETO-BARRIGA, ${ }^{1}$ SAMUel \\ PINEDA, ${ }^{1}$ ÁNGEL REBOLLAR-ALVITER, ${ }^{2}$ JUAN M. CHAVARRIETA $^{1} \mathrm{Y}$ \\ JosÉ ISAAC FIGUEROA ${ }^{1 *}$ \\ ${ }^{1}$ Instituto de Investigaciones Agropecuarias y Forestales, Universidad Michoacana de San Nicolás de \\ Hidalgo, Km. 9.5 carretera Morelia-Zinapécuaro, Tarímbaro, Michoacán, 58880, México. \\ ${ }^{2}$ Universidad Autónoma Chapingo/Centro Regional Morelia, Michoacán, 58170, México. \\ *Autor de correspondencia: <figueroaji@yahoo.com.mx.>
}

Martínez, A. M., Barreto-Barriga, O., Pineda, S., Rebollar-Alviter, A., Chavarrieta, J. M. y Figueroa, J. I. 2014. Parasitoides asociados a los enrolladores de hojas de zarzamora Argyrotaenia montezumae Walsingham y Amorbia sp. (Lepidoptera: Tortricidae), en Michoacán, México. Acta Zoológica Mexicana (n.s.), 30(3): 553-563.

RESUMEN. Los enrolladores de hojas Argyrotaenia montezumae Walsingham y Amorbia sp. son especies de tortrícidos asociados a plantas de zarzamora, en Michoacán, México. En este estudio se analiza y compara la abundancia y diversidad de parasitoides asociados a estas especies de enrolladores en dos plantaciones comerciales en Los Reyes, Michoacán. Se recolectaron 1682 hojas tiernas infestadas con larvas de enrolladores, de donde se obtuvieron 994 ejemplares de cuatro especies de parasitoides. Los parasitoides obtenidos fueron Chelonus sp. (41.6\%), Apanteles n. sp. (34.2\%), Colpoclypeus michoacanensis Sánchez y Figueroa (23.1\%) y Bracon sp. (1\%). Apanteles n. sp. registró el porcentaje de parasitismo más alto (26.9\%) y el que con mayor periodicidad se presentó.

Palabras clave: Zarzamora, tortricidos, parasitismo natural, periodicidad, control biológico

Martínez, A. M., Barreto-Barriga, O., Pineda, S., Rebollar-Alviter, A., Chavarrieta, J. M. \& Figueroa, J. I. 2014. Parasitoids associated with the blackberry leafrollers Argyrotaenia montezumae Walsingham and Amorbia sp. (Lepidoptera: Tortricidae), in Michoacan, Mexico.Acta Zoológica Mexicana (n.s.), 30(3): 553-563.

ABSTRACT. The leafrollers Argyrotaenia montezumae Walsingham and Amorbia sp. are tortricids associated with blackberry plants, in Michoacan, Mexico. In this study we analyzed and compared the abundance and diversity of parasitoids associated with these leafroller species in two commercial plantings in Los Reyes, Michoacan. A total of 1682 young leaves infested with leafroller larvae were collected, of these 994 specimens of four parasitoid species were obtained. The parasitoids were Chelonus

Recibido: 12/12/2013; aceptado: 27/05/2014. 
sp. (41.6\%), Apanteles n. sp. (34.2\%), Colpoclypeus michoacanensis Sánchez \& Figueroa (23.1\%), and Bracon sp. (1\%). Apanteles n. sp. recorded the highest percent of parasitism (26.9\%) and it was also the species that appeared more frequently.

Key words: blackberry, tortricids, natural parasitism, periodicity, biological control.

\section{INTRODUCCIÓN}

Los frutos de zarzamora, Rubus sp., son apreciados por su alto valor nutritivo, color, aroma y sabor. En México, el cultivo de esta frutilla representa una derrama económica muy importante debido a que genera miles de empleos directos en su producción y comercialización (Sánchez 2008). Entre los años 2001 al 2011 el área dedicada a este cultivo se incrementó exponencialmente de 997 a 11296 ha, lo que derivó en un aumento de su producción de 11569 a 135562 ton (SIAP 2012). Entre los principales estados productores de zarzamora destacan Michoacán, Guanajuato, Hidalgo y Estado de México, concentrándose en Michoacán el 97\% de la producción total nacional (Sánchez 2008). En 2011, en este estado se sembraron 10752 ha de zarzamora, de donde se obtuvo una producción de 129403 ton y una captación de divisas de \$3,490,097 (SIAP 2011). Desafortunadamente, en este cultivo se registran los enrolladores de hojas Argyrotaenia montezumae y Amorbia sp., cuyas larvas se alimentan de las hojas tiernas en formación y su presencia es inconfundible debido a que las larvas doblan las hojas, para alimentarse y protegerse, con seda que ellas mismas producen (Barreto 2012).

En el estado de Michoacán, el control implementado contra estos enrolladores de hojas está basado principalmente en insecticidas sintéticos de amplio espectro (Sánchez 2008). Sin embargo, esta medida puede reducir las poblaciones nativas de enemigos naturales, contaminar el medio ambiente y provocar el desarrollo de resistencia de estos insectos fitófagos. Por esta razón surge la necesidad de buscar alternativas ecológicas, tales como el uso de parasitoides, que sean efectivas contra estos insectos y que además sean compatibles con las prácticas de manejo integrado de plagas. Al respecto, sólo se conocen los trabajos de López (2009) y Juárez et al. (2010), en los cuales se menciona la presencia de parasitoides que atacan al enrollador de hojas de zarzamora A. montezumae, pero no proporcionan datos biológicos. Por lo tanto, en el presente trabajo se realizaron recolectas sistematizadas en el cultivo de zarzamora con el propósito de conocer la periodicidad de las especies de parasitoides, así como determinar los porcentajes de parasitismos natural que ejercen sobre las larvas de estos enrolladores.

\section{MATERIAL Y MÉTODOS}

Sitio de trabajo. Este trabajo se realizó en dos plantaciones de zarzamora variedad Tupy, una abandonada en el Rancho Huatarillo y una con manejo convencional en el Rancho El Guayabo, en el estado de Michoacán (Cuadro 1). Ambas plantaciones 
Cuadro 1. Sitios de estudio, fechas de recolecta y número de larvas recolectadas en el estado de Michoacán, México.

\begin{tabular}{|c|c|c|c|}
\hline Sitios de estudio & Municipio & $\begin{array}{r}\text { Fecha de rec } \\
\text { de larva }\end{array}$ & $\begin{array}{l}\text { número } \\
\text { idas }\end{array}$ \\
\hline $\begin{array}{l}\text { Huatarillo } \\
19^{\circ} 34^{\prime} 07^{\prime \prime} \mathrm{N}, 102^{\circ} 27^{\prime} 37^{\prime \prime} \mathrm{O}, 1,350 \mathrm{~m}\end{array}$ & Peribán & $\begin{array}{c}20-\mathrm{VIII}-2010 \\
28-\mathrm{VIII}-2010 \\
11-\mathrm{IX}-2010 \\
25-\mathrm{IX}-2010 \\
16-\mathrm{X}-2010 \\
6-\mathrm{XI}-2010 \\
27-\mathrm{XI}-2010\end{array}$ & $\begin{array}{c}24 \text { larvas } \\
104 \\
299 \\
156 \\
39 \\
136 \\
115\end{array}$ \\
\hline $\begin{array}{l}\text { El Guayabo } \\
19^{\circ} 34^{\prime} 31^{\prime \prime} \mathrm{N}, 102^{\circ} 27^{\prime} 46^{\prime \prime} \mathrm{O}, 1351 \mathrm{~m}\end{array}$ & Los Reyes & $\begin{array}{c}20-\mathrm{VIII}-2010 \\
28-\mathrm{VIII}-2010 \\
11-\mathrm{IX}-2010 \\
25-\mathrm{IX}-2010 \\
16-\mathrm{X}-2010 \\
6-\mathrm{XI}-2010 \\
27-\mathrm{XI}-2010\end{array}$ & $\begin{array}{c}175 \text { larvas } \\
141 \\
85 \\
97 \\
169 \\
142 \\
0\end{array}$ \\
\hline
\end{tabular}

de 10 meses de edad, $1.70 \mathrm{~m}$ de altura y con una distancia de siembra de $40 \mathrm{~cm}$ entre plantas y 2 m entre hileras. La primera plantación tenía cuatro meses abandonada, mientras que la segunda su manejo convencional consistió en actividades de fertilización, poda, defoliación, estimulación y desarrollo de brotes laterales fructificantes, así como aplicaciones de los insecticidas malatión, diazinon, cipermetrina, z-cipermetrina, spinosad, spinetoram y Bacillus thuringiensis para el control de enrolladores de hojas y trips; en lo sucesivo ambos sitios serán referidos como Huatarillo y El Guayabo. Entre agosto y noviembre de 2010, correspondiente al segundo ciclo productivo del cultivo, se recolectaron un total de siete muestras por sitio, aproximadamente una cada 15 días. Se eligió por sitio de estudio y fecha una superficie de 1 ha de cultivo, cuyos surcos se recorrieron en forma de zig zag para recolectar las hojas tiernas con signos de enrollamiento. Las hojas se colocaron en contenedores de plástico de $26 \mathrm{x}$ 18 x $4.5 \mathrm{~cm}$ y se trasladaron al laboratorio de entomología (LE) del Instituto de Investigaciones Agropecuarias y Forestales (IIAF) de la Universidad Michoacana de San Nicolás de Hidalgo (UMSNH), en Morelia, Michoacán. Cada hoja se acondicionó de manera individual en caja Petri de plástico ( $32 \times 22 \times 6 \mathrm{~cm}$ ) y se mantuvo a $25 \pm 2{ }^{\circ} \mathrm{C}$, $60 \pm 5 \%$ de humedad relativa y un fotoperiodo de 16:8 h (luz/oscuridad). Para evitar la deshidratación de los mismos se les colocó un algodón húmedo en su base cada dos días. El desarrollo de las larvas se siguió hasta la obtención de adultos.

Determinación taxonómica, parasitismo y periodicidad de los parasitoides. La determinación taxonómica se realizó por personal del Centro Nacional de Referencia Fitosanitaria de la Dirección General de Sanidad Vegetal en Distrito Federal, México 
(CNRF-DGSV) y Antonio Vives (Sociedad Hispano-Luso-Americana de Lepidopterología, España), determinando a Argyrotaenia montezumae y Amorbia sp. Debido a la similitud de enrollamiento de hojas que provocan las larvas de ambas especies de insectos fue difícil diferenciarlas en estado larval, por lo mismo los parasitoides emergidos no se pudieron asociar a una especie de enrollador en particular, por ello se consideró como si hubieran parasitado una sola especie. Los porcentajes de parasitismo se determinaron de la siguiente manera: en parasitoides solitarios, el parasitismo se determinó con base al número de parasitoides emergidos entre el total de larvas recolectadas por muestra. En el caso de los parasitoides gregarios se consideró el número de larvas de enrolladores de hojas parasitadas entre el total de larvas recolectadas por muestra. La periodicidad de cada especie de parasitoide se determinó con sus emergencias en cada fecha de recolecta. En especies gregarias, el cálculo del promedio de individuos que emergieron por larva parasitada se determinó contabilizando todos los ejemplares emergidos de una misma especie de parasitoide entre el número de larvas que parasitaron. Todos los parasitoides emergidos se colocaron en frascos con alcohol al 70\% y se procesaron para su preservación y montaje. La determinación taxonómica de los parasitoides se realizó con Muesebeck (1920), Mason (1974), Whitfield (1997), Shaw (1997) y Sánchez et al. (2011). Todos los ejemplares se depositaron en la colección de "Himenóptera Parasítica” del IIAF-UMSNH.

\section{RESULTADOS}

Se recolectaron 1682 larvas de diferentes estadios de enrolladores de hojas, 873 se obtuvieron de Huatarillo y 809 de El Guayabo (Cuadro I). En el primer sitio se contabilizaron 249 larvas parasitadas y en el segundo 167.

Diversidad de parasitoides. Se obtuvieron 994 ejemplares de cuatro especies de parasitoides, tres pertenecen a la familia Braconidae: Chelonus sp. (41.6\%), Apanteles n. sp. (34.2\%) y Bracon sp. (1\%) y una corresponde a la familia Eulophidae: Colpoclypeus michoacanensis Sánchez \& Figueroa (23.1\%). Las cuatro especies fueron recolectadas en ambos sitios, aunque en Huatarillo se registró 1.7 veces más parasitoides emergidos que en El Guayabo (Cuadro 2). Dos especies tienen hábitos solitarios, Apanteles n. sp. y Bracon sp., mientras que Chelonus sp. y C. michoacanensis mostraron ser gregarias. La presencia de estos últimos provocó que el número total de larvas parasitadas fuera diferente al número total de parasitoides emergidos. Por ejemplo, en Huatarillo los 221 individuos de Chelonus sp. y 195 de C. michoacanensis emergieron de 23 y 17 larvas de enrolladores, respectivamente. En el caso de El Guayabo, los 193 individuos de Chelonus sp. y 35 de C. michoacanensis emergieron respectivamente de 21 y 5 larvas de enrolladores. En términos generales, las especies gregarias Chelonus sp. y C. michoacanensis presentaron una emergencia promedio de nueve y 10 individuos por larva parasitada, respectivamente. 
Cuadro 2. Número y especies de parasitoides emergidos por localidad estudiada.

\begin{tabular}{|c|c|c|c|c|c|}
\hline \multirow{2}{*}{\multicolumn{3}{|c|}{ Parasitoides }} & Huatarillo & El Guayabo & \multirow[b]{2}{*}{ Total (\%) } \\
\hline & & & (n) & (n) & \\
\hline Familia & Especie & Hábito & & & \\
\hline \multirow[t]{3}{*}{ Braconidae } & Apanteles sp. & Solitario & 200 & 140 & $340(34.2 \%)$ \\
\hline & Bracon sp. & Solitario & 9 & 1 & $10(1.0 \%)$ \\
\hline & Chelonus sp. & Gregario & 221 & 193 & $414(41.6 \%)$ \\
\hline \multirow[t]{2}{*}{ Eulophidae } & Colpoclypeus michoacanensis & Gregario & 195 & 35 & $230(23.1 \%)$ \\
\hline & & & 625 & 369 & 994 \\
\hline
\end{tabular}

n, número de individuos

Parasitismo natural y periodicidad. De acuerdo a este estudio, Apanteles n. sp. fue el parasitoide principal de los enrolladores de hoja en zarzamora, debido a que emergió en prácticamente todas las fechas de muestreo y ejerció los valores más altos de parasitismo (Fig. 1a). En Huatarillo el porcentaje de parasitismo más alto fue de 26.9\%, mientras que en El Guayabo fue de 22\%. La figura 1a ilustra una coincidencia en los picos más altos de parasitismo que tuvieron ambos sitios de estudios, el cual correspondió a la fecha del 28 de agosto. En el trascurso de todas las recolectas se obtuvieron especímenes de Apanteles n. sp., lo que indicó que estuvo presente en la zarzamora desde agosto a noviembre. Además ejerció el porcentaje de parasitismo más elevado (26.9\%), comparado con Bracon sp. (2.7\%, Fig. 1b), Chelonus sp. (6.5\%, figura 1c) y C. michoacanensis (3.8\%, Fig. 1d). Bracon sp. sólo se encontró en dos muestras de Huatarillo y una de El Guayabo. En Huatarillo ocurrió en las muestras del 11 de septiembre ( $2.7 \%$ de parasitismo) y 6 de noviembre $(0.7 \%$ de parasitismo), mientras que en el Guayabo solo se presentó en la muestra del mes de agosto ( $0.7 \%$ de parasitismo). Chelonus sp., a diferencia de las otras, fue la única especie que ejerció el porcentaje de parasitismo más alto en El Guayabo, debido a que las tres especies restantes lo hicieron en Huatarillo. En el Guayabo el porcentaje de parasitismo más alto correspondió a 6.5\%, mientras que en Huatarillo fue de 4.7\% (Fig. 1c). En el Guayabo no hubo presencia de Chelonus sp. en las recolectas del 28 de agosto y del 27 de noviembre, mientras que en Huatarillo no hubo presencia en las recolectas del 20 de agosto y 6 de noviembre, lo que quiere decir que las poblaciones de Chelonus sp. están presentes desde septiembre hasta noviembre en las dos localidades, independientemente que no se encuentren en muestras intermedias para una u otra localidad. El eulófido C. michoacanensis se encontró parasitando a los enrolladores de hojas en los dos sitios de estudio (Fig. 1d), pero en Huatarillo se registró el porcentaje de parasitismo más alto (3.8\%), comparado con el más alto de El Guayabo (2.1\%). En Huatarillo se presentó en dos muestras del mes de septiembre y una muestra de noviembre, mientras que en El Guayabo estuvo presente en una muestra de 


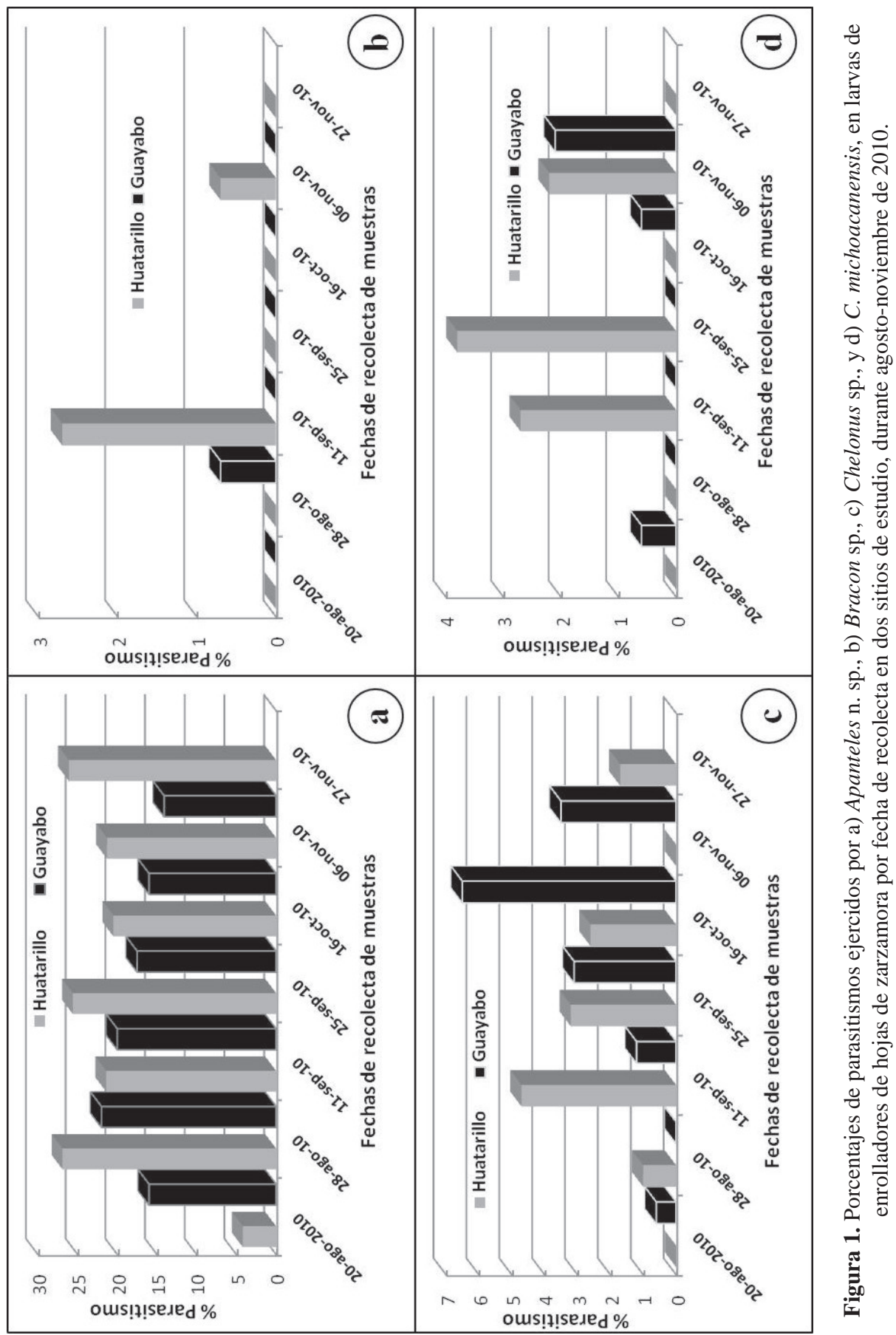


agosto, de octubre y noviembre, lo que indica que C. michoacanensis tiene presencia en el cultivo de zarzamora desde agosto a noviembre, independientemente de que no se observó presencia en muestras intermedias.

\section{DISCUSIÓN}

Los resultados obtenidos en el presente estudio muestran que Huatarillo tuvo más abundancia de parasitoides que El Guayabo, diferencia que puede ser explicada a que Huatarillo fue una parcela abandonada y sin aplicaciones de insecticidas. Información sobre parasitoides asociados con enrolladores de hojas en el cultivo de zarzamora es limitada. Algunos trabajos similares que tuvieron como propósito conocer especies de parasitoides asociados a tortrícidos, donde también se hicieron comparaciones entre dos tipos de huertos (orgánicos y convencionales), fueron realizados en Columbia Británica (Canadá) y California (E.U.A.). El primero fue conducido por Li et al. (1999) con el "enrollador de frambuesa”, Choristoneura rosaceana (Harris). En este estudio se registraron 14 especies de parasitoides (13 himenópteros y 1 díptero), de los cuales el bracónido Macrocentrus nigridorsis Viereck fue el más abundante. Los mismos autores también resaltan que en parcelas abandonadas encontraron los más altos porcentajes de parasitismos ( 30\%), similar a lo que ocurrió en el presente estudio. El segundo trabajo fue realizado por Walker \& Welter (2004), cuyo propósito fue también conocer la diversidad de parasitoides que atacaron al "enrollador de hojas del manzano", Argyrotaenia citrana (Fernald). Estos últimos autores reportaron a una especie de Apanteles como la más abundante, similar a lo que se encontró en nuestro estudio. De los dos estudios citados, sólo Li et al. (1999) reportaron a un parasitoide gregario, Macrocentrus nigridorsis Viereck, cuya emergencia promedio por larva parasitada fue de casi 36 individuos. Otros trabajos que registraron parasitoides de enrolladores fueron realizados por Kido et al. (1981), quienes encontraron en el cultivo de la vid, Vitis vinifera L., cuatro especies de parasitoides que atacaron a A. citrana. De la misma manera, Wilkinson et al. (2004) registraron en huertos de manzanos a 20 especies de parasitoides que se asociaron a Ch. rosaceana. En México, específicamente en los municipios de Guerrero y Cuauhtémoc, en Chihuahua, Quintana (2010) reportó en huertos de manzanos a siete especies de parasitoides que atacaron a los enrolladores Ch. rosaceana y Argyrotaenia sp.

En el presente estudio no saber diferenciar a los enrolladores $A$. montezumae y Amorbia sp. complicó conocer de qué especie de enrollador emergieron los parasitoides. Esto mismo ocurrió en el trabajo de Cossentine et al. (2004), quienes en huertos de manzano con manejo orgánico no pudieron asociar las larvas de los enrolladores Ch. rosaceana y Pandemis limitata (Robinson) con sus respectivos parasitoides.

La identidad taxonómica a nivel de especie de los ejemplares de Apanteles emergidos en este estudio aún se encuentra en proceso, ya que se trata de una especie no 
descrita (Sanchez et al. sin publicar). Apanteles n. sp. se encontró durante todo el periodo de estudio y ejerció los más altos porcentajes de parasitismos (arriba del 20\%) en las dos sitios estudiados. Cossentine et al. (2004) mencionan que las especies de parasitoides son estacionales, ya que en un periodo determinado de tiempo algunas especies aparecen con mayor frecuencia, mientras que en un periodo diferente la abundancia de las mismas se revierte, lo cual se atribuye a la disponibilidad del hospedero principal y a la existencia de hospederos alternos, ya que estos últimos incrementan la abundancia de otras especies de parasitoides. Entre los pocos trabajos que citan a especies de Apanteles que atacan a enrolladores tortrícidos destacan Walker \& Welter (2004), quienes citan a A. aristoteliae sobre A. citrana en huertos de manzanos de California, EUA. En México, Quintana (2010) también reporta a Apanteles sp. en huertos de manzanos atacando a Ch. rosaceana y Argyrotaenia sp.

Los ejemplares de Bracon sp. tampoco se determinaron a nivel de especie, solo se conoció que se trató de una especie solitaria. Quintana (2010) reportó a una especie gregaria de Bracon que atacó al "enrollador de hojas de manzana”, Ch. rosaceana. Así mismo, Milonas (2005) citó a Bracon hebetor (Say) como una importante especie gregaria que atacó al tortrícido Adoxophyes orana (Fischer von Röslerstamm). Respecto a los ejemplares de Chelonus, ellos se ubicaron en el subgénero Microchelonus, sin determinarse a nivel de especie. Hasta ahora no hay un estudio que cite alguna especie de Microchelonus que ataque a tortrícidos enrolladores. En esta investigación, Chelonus sp. fue la segunda especie más importante, dado que ejerció el segundo valor más alto de parasitismo (6.5\%), después de Apanteles n. sp. (que obtuvo 26.9\%). Se desconoce la razón del por qué se registró el porcentaje de parasitismo más alto en la parcela con manejo convencional, sin embargo pudiera considerarse como un indicio de que Chelonus sp. podría adaptarse al manejo convencional que se proporciona a las parcelas de zarzamora.

De los ejemplares de C. michoacanensis se desconocía mucho sobre su biología pero en este escrito se proporcionan más datos adicionales. Previo al año 2010, Colpoclypeus florus era la única especie reconocida a nivel mundial dentro del género, pero Sánchez et al. (2011) describieron del estado de Michoacán a C. michoacanensis como la segunda especie en el género. Los porcentajes de parasitismo que ejerció $C$. michoacanensis en los dos sitios de estudio resultaron ser muy bajos (0.6 a 3.8\%), sin embargo habría que recordar que el número total de larvas recuperadas por muestra correspondieron a dos especies de enrolladores, lo que posiblemente hizo que el porcentaje de parasitismo disminuyera. Por su parte, C. florus parece tener hasta un 95\% de parasitismo en diversos cultivos con problemas de tortrícidos de Europa, donde representa el principal agente de control biológico (Evenhuis 1974, Gruys \& Vaal 1984, van Veen \& Wijk 1987). En cuanto al número de individuos que emergen por larva parasitada, C. michoacanensis también presenta datos diferentes a lo reportado con C. florus. El número de individuos emergidos en $C$. florus es por arriba de los 50 
ejemplares en promedio por larva parasitada (Brunner 1996, Dijkstra 1986), en cambio en C. michoacanensis fue entre 5 a 30 individuos. En lo que se refiere a la periodicidad de esta especie de parasitoide, es lógico suponer que ellos aparecerán conforme existan hospederos disponibles, como sucede con C. florus. Pfannenstiel et al. (2010) realizaron en los estados de Washington y Oregon un estudio detallado con C. florus sobre la utilización de hospederos alternantes en ausencia de los hospederos potenciales. Al parecer, $C$. florus parasita a sus principales hospederos, Ch. rosaceana y $P$. pyrusana Kearfott (Lepidoptera: Tortricidae) en huertos de manzanos, en los meses de abril a julio (Pfannenstiel \& Unruh 2002), mientras que en los periodos donde no existen estos hospederos (septiembre a noviembre) utilizan a hospederos alternos como Ancylis comptana (Froelich), Xenotemna pallorana (Robinson) y Syndemis sp. (Tortricidae), Filatima sp. (Gelechiidae) y Caloptilia burgessiellia (Zeller) (Gracillariidae) (Pfannenstiel et al. 2010). Esta situación muestra claramente que C. florus parasita a diversos hospederos en un amplio periodo de tiempo, el cual va desde abril a noviembre; en cambio $C$. michoacanensis, en este estudio únicamente se encontró desde agosto a noviembre y únicamente atacando a las dos especies de enrolladores de hojas. En conclusión, de las cuatro especies de parasitoides encontradas, Apanteles n. sp. destacó con los más altos porcentajes de parasitismos y mayor periodicidad en el trascurso de la investigación, aunque se necesitan estudios adicionales para conocer más detalles de su biología, comportamiento y tolerancia a insecticidas.

AGRADECIMIENTOS. A la Coordinación de la Investigación Científica-UMSNH y Fundación Produce Michoacán por el financiamiento otorgado.

\section{LITERATURA CITADA}

Barreto-Barriga, O. 2012. Parasitoides de dos enrolladores de hojas de zarzamora con énfasis en la biología de Argyrotaenia montezumae (Walsingham) (Lepidoptera: Tortricidae) y de su parasitoide Apanteles cercana a aristoteliae (Viereck) (Hymenoptera: Braconidae). Tesis de Maestría. Programa Institucional de Maestría en Ciencias Biológicas, Universidad Michoacana de San Nicolás de Hidalgo. 96 pp.

Brunner, J. F. 1996. Discovery of Colpoclypeus florus (Walker) (Hymenoptera: Eulophidae) in apple orchards of Washington. The Pan Pacific Entomologist, 72: 5-12.

Cossentine, J., Jensen, L., Deglow, E., Bennett, A., Goulet, H., Huber, J. \& O’Hara, J. 2004. The parasitoid complex affecting Choristoneura rosaceana and Pandemis limitata in organically managed apple orchards. Biocontrol, 49: 359-372.

Dijkstra, L. J. 1986. Optimal selection and exploitation of hosts in the parasitic wasp Colpoclypeus florus (Hym., Eulophidae). Netherlands Journal of Zoology, 36: 177-301.

Evenhuis, H. H. 1974. Colpoclypeus florus (Hymenoptera: Eulophidae), an important potential parasite of Adoxophyes orana (Lepidoptera: Tortricidae) in apple orchards. Mededelingen van Faculteit van de Landbouwwetenschappen, Rijksuniversiteit Gent, 39: 769-775.

Gruys, P. \& Vaal, F. 1984. Colpoclypeus florus, an eulophid parasite of tortricids in orchards: Rearing, biology and use in biological control. Entomologia Experimentalis et Applicata, 36: 31-35. 
Juárez, G. A. C., Pineda, S., Martínez, A. M., Sánchez-García, J. A., Rebollar-Alviter, Á., LópezCruz, I., Coronado-Blanco, J. M. \& Figueroa, J. I. 2010. Parasitoides asociados a un enrollador de hojas del cultivo de zarzamora, en dos localidades del estado de Michoacán. 3er Encuentro nacional sobre ciencia, tecnología e innovación en México durante la última década: una visión crítica al futuro y 6to. Congreso Estatal de Ciencia y Tecnología. Michoacán.

Kido, H., Flaherty, D. L., Kennett, C. E., McCalley, N. F. \& Bosch, D. F. 1981. Seeking the reasons for differences in orange tortrix infestations. California Agriculture, 35: 27-28.

Li, S. Y., Fitzpatrick, S. M., Troubridge, J. T., Sharkey, M. J., Barron, J. R. \& O’Hara, J. E. 1999. Parasitoids reared from the obliquebanded lefroller (Lepidoptera: Tortricidae) infesting raspberries. The Canadian Entomologist, 131: 399-404.

López, C. I. 2009. Identificación, dinámica poblacional y parasitoides de dos lepidópteros plaga de zarzamora, Rubus fruticosus L., en tres regiones productoras del estado de Michoacán. Tesis de licenciatura. Facultad de Biología. Universidad Michoacana de San Nicolás de Hidalgo. Morelia, Michoacán, México. 61 pp.

Mason, W. R. M. 1974. The Apanteles species (Hymenoptera: Braconidae) attacking Lepidoptera in the micro-habitat of the spruce budworm (Lepidoptera: Tortricidae). The Canadian Entomologist, 106: $1087-1102$.

Milonas, P. G. 2005. Influence of initial egg density and host size on the development of the gregarious parasitoid Bracon hebetor on the different host species. BioControl, 50: 415-428.

Muesebeck, C. F. W. 1920. A revision of the north american species of Ichneumon-flies belonging to the genus Apanteles. Proceedings of the United States National Museum, 58(2349): 483-576.

Pfannenstiel, R. S. \& Unruh, T. R. 2002. Conservation of leafroller parasitoids through provision of alternate hosts in near-orchard habitats. In: Van Driesche R.G. (Ed.). Proceedings of the International Symposium on Biological Control of Arthropods. Honolulu, Hawaii, 14-18 Jan. 2002. USDA, Forest Service; pp. 256-262.

Pfannenstiel, R. S., Unruh T. R. \& Brunner, J. F. 2010. Overwintering hosts for the exotic leafroller parasitoid, Colpoclypeus florus: Implications for habitat manipulation to augment biological control of leafrollers in pome fruits. Journal of Insect Science, 10: 1-13.

Quintana, L. E. 2010. Parasitoides de enrolladores de la hoja (Lepidoptera: Tortricidae) del manzano en Chihuahua. pp. 145-148. In: A. V. M. Coria, B. N. Lara C., G. Orozco G., H. J. Muñoz F. y R. Sánchez, M. (Eds.). Memorias del XXXIII Congreso Nacional de Control biológico. Uruapán, Michoacán.

Sánchez, R. G. 2008. La red de valor de la zarzamora, El cluster de Los Reyes, Michoacán un ejemplo de reconversión competitiva. Fundación Produce Michoacán, A.C. 1ra ed. Morelia, Michoacán, México. Pp. 1-106.

Sánchez, G. J. A., Pineda, S., Martínez, A. M., Rebollar-Alviter, A., Juárez-Gutiérrez, A. C., López-Cruz, I. \& Figueroa, J. I. 2011. A new species of Colpoclypeus Lucchese (Hymenoptera: Eulophidae) from Mexico. Zootaxa, 2830: 64-68.

Sánchez, G. J. A., Figueroa, J. I., Whitfield, J., Pineda, S. \& Martínez, A. M. (En preparación). A new species of Apanteles Foerster (Hymenoptera: Braconidae, Microgastrinae) from Mexico.

Servicio de información Agroalimentaria y Pesquera (SIAP). 2011. Anuario estadístico de la producción agrícola. http://www.siap.gob.mx [Accesada el 19 de junio de 2013].

Servicio de información Agroalimentaria y Pesquera (SIAP). 2012. Anuario estadístico de la producción agrícola. http://www.siap.gob.mx [Accesada el 19 de junio de 2013].

Van Veen, J. C. \& Wijk, M. L. E. 1987. Parasitization strategy in the non-paralyzing ectoparasitoid Colpoclypeus florus (Hym., Eulophidae) towards its common summer host Adoxophyes orana (Lepidoptera: Tortricidae). Journal of Applied Entomology, 104: 402-417. 
Walker, K. R. \& Welter, S. C. 2004. Biological control potential of Apanteles aristoteliae (Hymenoptera: Braconidae) on populations of Argyrotaenia citrana (Lepidoptera: Tortricidae) in California apple orchards. Environmental Entomology, 33: 1327-1334.

Whitfield, J. B. 1997. Subfamily Microgastrinae. pp. 333-364. In: R. A. Wharton, P. M. Marsh \& M. J. Sharkey (Eds). Manual of the new world genera of the family Braconidae (Hymenoptera). International Society of Hymenopterists. Special Publication No. 1

Wilkinson, T. K., Douglas, A. L. \& Larry, J. G. 2004. Parasitism of Obliquebanded Leafroller (Lepidoptera: Tortricidae) in commercially managed Michigan apple orchards. Journal Economic of Entomology, 97: 1524-1530. 